\title{
Ausmaß und mögliche Konsequenzen der Arbeitsmigration im Rahmen des GATS für die Länder der Europäischen Union
}

Die Diskussion um die Dienstleistungsrichtlinie in der Europäischen Union hat die politische Brisanz der Liberalisierung des Dienstleistungssektors deutlich gemacht. Diese Debatte bezog sich ausschließlich auf die innereuropäische Regulierung und nahm die möglichen Auswirkungen des Allgemeinen Übereinkommens über den Dienstleistungsverkehr (GATS) auf die Dienstleistungsarbeit in der EU wenig oder gar nicht zur Kenntnis. Der Beitrag schließt diese Lücke und fragt danach, welche Auswirkungen das GATS-Abkommen im Rahmen der Welthandelsorganisation (WTO) für die Länder der Europäischen Union hat. Welche Konsequenzen ergeben sich daraus für die Arbeitsmärkte in der EU? 1

\section{Einleitung}

Die zeitlich befristete Grenzüberschreitung von Dienstleistungserbringern in der Erbringungsart 4 des Allgemeinen Übereinkommens über den Dienstleistungsverkehr (GATS) macht lediglich einen kleinen Teil des internationalen Dienstleistungshandels wie auch der Grenzüberschreitungen von Arbeitskräften insgesamt aus. Und doch kristallisierte sich die Erbringungsart 4 (Modus 4) des GATS in den Verhandlungen der Entwicklungsrunde von Doha als eines der Hauptthemen heraus. Die Diskussionen über die zeitlich begrenzte Anwesenheit von Dienstleistungserbringern im Ausland spielen sich vor dem allgemeinen Hintergrund zunehmender Arbeitsmigration, der wachsenden Bedeutung des Dienstleistungssektors sowie des internationalen Dienstleistungshandels ab. Aus Sicht der EU sind die Debatten um Modus 4 auch eng verflochten mit anderen Diskussionen: der Debatte um die EU-Erweiterung und um die erhöhte Mobilität der Arbeitskräfte aus den neuen Mitgliedstaaten; um die Verlagerung von Kapital; um schleppendes Wachstum und hohe Arbeitslosigkeit in einigen Mitgliedstaaten sowie um die Dienstleistungsrichtlinie der EU.

Das Zusammenspiel verschiedenster Faktoren - darunter der Anstieg von Handels- und Investitionsflüssen, der Technologiewandel, rückläufige Transport- und Kommunikationskosten sowie globale Unternehmensketten - wird voraussichtlich die Forderungen nach der Liberalisierung des grenzüberschreitenden Verkehrs natürlicher Personen verstärken (OECD 2003a, 2003b). Die entwickelten Länder vollführen hier einen Balanceakt. Einerseits begünstigt die alternde Bevölkerung die Einwanderung, andererseits gibt es eine $\mathrm{Ab}$ wehrhaltung gegenüber einer dauerhaften Migration aus Drittländern. Dem steht das starke Interesse der Entwicklungsländer an einer weiteren Liberalisierung der Regelungen in Erbringungsart 4, insbesondere für Arbeitskräfte mit mittlerem und niedrigem Qualifikationsstand, gegenüber.

\section{GATS und die Erbringungsart 4}

\subsection{WAS IST DAS GATS?}

Das GATS schafft einen für alle WTO-Mitgliedstaaten gültigen Rechtsrahmen für den internationalen Dienstleistungshandel. Es wurde in der Uruguay-Runde der Welthandelsgespräche vereinbart und trat 1995 in Kraft. Ziel des Übereinkommens ist eine fortschreitende Liberalisierung des Dienstleistungshandels, die durch regelmäßige Verhandlungsrunden erreicht werden soll. Das Übereinkommen sieht vier Modi oder Erbringungsarten für den Handel mit Dienstleistungen zwischen WTOMitgliedern vor. Diese definieren sich nach der Herkunft von Dienstleistungserbringer und Verbraucher sowie nach der Form ihrer Anwesenheit im jeweiligen Hoheitsgebiet zum Zeitpunkt der Dienstleistungserbringung: Die Erbringungsarten werden dabei in vier Modi unterteilt - mode 1 (grenzüberschreitende Erbringung, wie z. B. die Leitweglenkung von Telefongesprächen über Call-Center), mode 2 (Nutzung von Dienstleistungen im Ausland), mode 3 (kommerzielle Präsenz im Ausland, wie z. B. die Einrichtung einer Niederlassung im Ausland; Eintritt in ein Gemeinschaftsunternehmen) und mode 4 - der Kernpunkt dieses Artikels -, die sich auf die zeitlich begrenzte Anwesenheit von Dienstleistungserbringern im Ausland bezieht.

Die Verhandlungen zum GATS beruhen auf einem System von Forderungen und Angeboten: Länder stellen Forderungen zur Liberalisierung des Handels mit Dienstleistungen an WTO-Partner. Zugleich machen sie Angebote, inwieweit sie zur Marktöffnung bereit sind. Nach dem ursprünglichen Zeitplan sollten die revidierten GATS-Angebote im Juli 2006 vor-

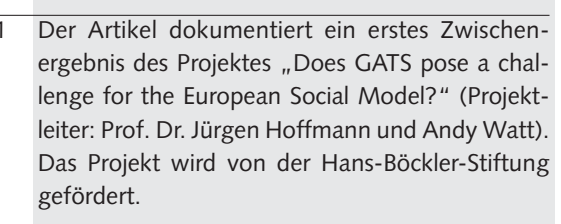
ergebnis des Projektes „Does GATS pose a challenge for the European Social Model?" (Projektleiter: Prof. Dr. Jürgen Hoffmann und Andy Watt). Das Projekt wird von der Hans-Böckler-Stiftung gefördert.

Gintare Kemekliene, M. A., Wissenschaftlerin am Europäischen Gewerkschaftsinstitut, Brüssel. Arbeitsschwerpunkte: Globalisierung, GATS und dessen Einfluss auf den Arbeitsmarkt. e-mail: gkemekliene@etui-rehs.org

Übersetzung aus dem Englischen: Silke Gebhard 
liegen, und die endgültigen Entwurfslisten der Verpflichtungen in dieser Runde sollten bis Oktober 2006 stehen (Sinclair 2006). Angesichts der vorläufigen Aufhebung der Welthandelsrunde von Doha wurden auch die Gespräche zur Dienstleistungsthematik ausgesetzt.

\subsection{BEGRIFFSBESTIMMUNG UND GELTUNGSBEREICH VON ERBRINGUNGSART 4}

Im GATS wird der Dienstleistungshandel nach der Erbringungsart 4 beschrieben als "die Erbringung einer Dienstleistung durch einen Dienstleistungserbringer eines Mitglieds mittels Präsenz natürlicher Personen eines Mitglieds im Hoheitsgebiet eines anderen Mitglieds". Sie erfasst den zeitlich begrenzten Aufenthalt natürlicher Personen, die in vier Kategorien unterteilt werden:

- Selbstständig Tätige (Freiberufler, die zum Zwecke der temporären Erbringung einer Dienstleistung in das Hoheitsgebiet eines anderen Mitgliedstaates einreisen);

- Innerbetrieblich Entsandte (Manager und Spezialisten, Nachwuchskräfte);

- Geschäftsreisende und Vertriebspersonal für Dienstleistungen, die in das Hoheitsgebiet eines anderen Mitglieds einreisen, um eine kommerzielle Präsenz einzurichten oder um über den Vertrieb von Dienstleistungen zu verhandeln;

- Vertragsdienstleister, die im Rahmen eines Vertrages zwischen ihrem Arbeitgeber und dem Konsumenten einer Dienstleistung im Hoheitsgebiet eines anderen Mitgliedstaates in dieses andere WTO-Mitgliedsland einreisen.

Das GATS umfasst generell jede internationale temporäre Migration von Dienstleistungserbringern gleich welcher Qualifikation. Des Weiteren gilt die Erbringungsart 4 im GATS für Staatsangehörige von WTOMitgliedstaaten und unter bestimmten Umständen auch für dauerhaft dort ansässige Personen, die im Ausland Dienstleistungen erbringen wollen. Die tatsächlichen Grenzen des Geltungsbereichs von Erbringungsart 4 sind jedoch nur sehr ungenau bestimmt. Das wirft verschiedene Probleme auf: Erstens besagt die Anlage zum GATS, dass die Erbringungsart 4 keine natürlichen Personen erfasst, die Zugang zum Arbeitsmarkt des Aufnahmelandes suchen; dass sie darüber hinaus nicht gilt für
Maßnahmen, die sich auf das Erlangen der entsprechenden Staatsbürgerschaft, den Aufenthalt oder die Beschäftigung auf dauerhafter Basis beziehen. Die Anlage besagt ferner, dass sie die Rechte der Staatsregierungen zur Regulierung von Einreise und vorübergehendem Aufenthalt nicht berührt, sofern diese Maßnahmen die Verpflichtungen aus GATS nicht zunichte machen oder schmälern. Die Erbringungsart 4 umfasst aber den Verkehr unabhängiger Dienstleistungserbringer, die eine befristete Beschäftigung im Ausland suchen. Die Definition von Erbringungsart 4 scheint also eine Frage der Interpretation zu sein.

Ein weiteres Problem ist darin zu sehen, dass die Erbringungsart 4 im GATS zwar Dienstleistungserbringer erfasst, die lediglich vorübergehend im Ausland verkehren, dass im Übereinkommen aber nicht definiert ist, was „vorübergehend“ bedeutet. Die gestattete Aufenthaltsdauer ist letztlich in den Angeboten der Mitglieder festgelegt und schwankt je nach der Art der Arbeit und normalerweise auch nach dem Qualifikationsgrad zwischen einigen Monaten und einigen Jahren (verlängerbar). Ferner bringen Kritiker des Übereinkommens eine Reihe weiterer spezifischer Beispiele für die Schwierigkeiten zur Sprache, ein einheitliches Konzept für Erbringungsart $4 \mathrm{zu}$ erarbeiten. So sei in der Kategorie der selbstständig Tätigen nicht klar, ob in lokalen Unternehmen angestellte Ausländer ebenfalls unter den Modus 4 fallen. Auch ist nicht durchschaubar, ob das GATS entsandte Arbeitnehmer erfasst und ob Zeitarbeiter, die landwirtschaftliche Dienstleistungen erbringen, im Rahmen des GATS vorübergehend im Ausland verkehren könnten. So kann man konstatieren, dass aufgrund der schlecht umrissenen Bestimmungen im GATS erst die realen Angebote und die in diesen realen Angeboten festgelegten Bedingungen und Einschränkungen die genauen Modalitäten klären.

\subsection{DAS VOLUMEN DER GEPLANTEN EU-VERPFLICHTUNGEN}

Generell sind Menge und Umfang der Verpflichtungen in der Erbringungsart $4 \mathrm{im}$ Vergleich zu den anderen Arten der Erbringung von Dienstleistungen begrenzt. Die Verpflichtungen sind im Allgemeinen eher horizontaler denn branchenspezifischer Natur - das heißt, sie gelten ohne Unterscheidung für alle Sektoren, die in der
Liste der Verpflichtungen eines Mitglieds aufgeführt sind. Sie beschränken sich zudem hauptsächlich auf innerbetrieblich Entsandte, wie leitende Angestellte und Fachkräfte, oft in Verbindung mit Erbringungsart 3 (kommerzielle Präsenz).

Die EU legte das ursprüngliche Angebot 2003 und das revidierte Angebot im Juni 2004 vor. Die Erbringungsart 4 wurde weiter geöffnet, indem zusätzliche Möglichkeiten der Grenzüberschreitung hochqualifizierter natürlicher Personen vorgesehen wurden. Im überarbeiteten EU-Angebot wurden die Zugangsbedingungen für viele Branchen erweitert; die neuen Mitgliedstaaten sind vielfältige Verpflichtungen eingegangen, um an das in der restlichen EU bereits gebotene Maß der Liberalisierung anzuschließen.

Unter der Kategorie der innerbetrieblich Entsandten (Manager, sogenannte Graduate Trainees und Spezialisten) werden Einreise und Aufenthalt für Manager und Spezialisten im EU-Angebot auf maximal drei Jahre begrenzt (Ausnahme Lettland: fünf Jahre); ferner können Nachwuchskräfte (Graduate Trainees) für Zeiträume von bis zu einem Jahr in die verbundenen Unternehmen in den 25 Staaten der EU versetzt werden. Geschäftsreisende können für maximal 90 Tage jährlich (Ausnahme Estland: innerhalb eines halben Jahres) in die EU einreisen und sich dort aufhalten, wenn sie über den Verkauf von Dienstleistungen verhandeln oder eine kommerzielle Präsenz gründen wollen. Vertragsdienstleistern (Kategorie: Contractual service suppliers, CSS), die in den 25 EU-Mitgliedstaaten keine kommerzielle Präsenz haben, ist es gestattet, in 21 Sektoren für maximal 6 Monate innerhalb eines Jahres Dienstleistungen zu erbringen; sie müssen dabei „den Gesetzen, Auflagen und Bestimmungen in der Europäischen Gemeinschaft und in den Mitgliedstaaten genügen, die Erfüllungsort des Vertrages sind" (Europäische Kommission 2003, eigene Übersetzung). In der Kategorie der selbstständig Tätigen (Kategorie: Independent Professional, IP) ist es selbstständigen Dienstleistern gestattet, bis zu einem Jahr lang Dienstleistungen in der EU in den Bereichen Recht, Architektur, Ingenieurwesen, Informationstechnik, Unternehmensberatung und Übersetzung zu erbringen.

Im revidierten EU-Angebot müssen die Dienstleister in den Kategorien CSS und IP (Vertragsdienstleister und selbstständig Tätige) über einen Universitätsabschluss 
verfügen bzw. über den Nachweis fachlicher Qualifikationen auf entsprechendem Niveau. Fachqualifikationen und mindestens drei Jahre Erfahrung im Sektor sind für die Vertragsdienstleister gefordert, und sechs Jahre in der Branche für selbstständig Tätige. Ferner unterliegen die Verpflichtungen für die Kategorien CSS und IP außer in Dänemark, den Niederlanden, in Schweden und im Vereinigten Königreich numerischen Obergrenzen. Darüber hinaus gelten in Polen für selbstständig tätige Freiberufler und in Italien für Vertragsdienstleister keine numerischen Obergrenzen. Für die neuen Mitgliedstaaten in Mittel- und Osteuropa (CEE) sollen Verpflichtungen in diesen Kategorien voraussichtlich ab Januar 2011 in Kraft treten.

Anhand der Angebote allein lässt sich jedoch nur schwer feststellen, wie umfassend die Liberalisierung tatsächlich sein wird. Bis dato gelten die Angebote als Ausdruck eines „Stillstands“ in dem Sinne, dass die angebotenen Verpflichtungen die existierende Situation lediglich widerspiegeln und keine Erweiterungen in Aussicht gestellt werden. Auch sind die vorgelegten Angebote insofern vorläufiger Natur, als die Verhandlungen über ihre Inhalte erst anlaufen müssen. Es fehlt somit noch an einer Bewertungsgrundlage für die in Erbringungsart 4 vorgesehenen Liberalisierungen.

\subsection{DIE INTERESSENLAGEN IN DEN VERHANDLUNGEN ÜBER ERBRINGUNGSART 4}

In den Verhandlungen über die vorübergehende Migration von Dienstleistungserbringern nach Erbringungsart 4 sind verschiedene Interessen im Spiel. Die Befürworter der Übereinkunft (namentlich die WTO, die Europäische Kommission, das European Services Forum und die OECD) heben auf die Vorteile einer weiteren Liberalisierung ab. Die Interessengruppen, die große internationale Dienstleistungsunternehmen in der EU vertreten, fordern weitere Verpflichtungen in der Erbringungsart 4, insbesondere im Hinblick auf Facharbeiter (European Service Forum 2005). Des Weiteren betonen sie ein strategisches Interesse an der weiteren Liberalisierung für Personen, die eine kommerzielle Präsenz in einem anderen Land anstreben (innerbetrieblich Entsandte und Geschäftsreisende). Dies wird zur Festigung bestehender und künftiger spezifischer Verpflichtungen in Erbringungsart 3 als wichtig erachtet wenn etwa ein ausländisches Dienstleistungsunternehmen eine kommerzielle Präsenz in einem Drittland aufbauen will, könnten nach Erbringungsart 4 Zeitarbeiter vor, während und nach dem Investitionsprozess einreisen. Man sieht die Erbringungsart 4 ferner als Zugangserleichterung für höher qualifizierte und produktivere Erwerbstätige und als eine Möglichkeit, um Schwankungen in der Nachfrage nach Dienstleistungen auszugleichen. Aus geschäftlicher Warte sollen globale Gesellschaften in der Lage sein, Mitarbeiter und Mitarbeiterinnen bedarfsorientiert an andere Standorte zu versetzen. Die Wirtschaftslobby hat Interesse an der Einführung des GATS-Visums: Es würde weniger stringente Zugangs- und Aufenthaltsregelungen schaffen; es würde sowohl für horizontale wie auch für sektorale Verpflichtungen gelten; und es würde für die jeweiligen Arbeitnehmerkategorien separate Bedingungen enthalten (OECD 2003c).

Viele Staatsregierungen, Sozialpartner und einige nichtstaatliche Organisationen in der EU haben Bedenken bezüglich einer weiteren Liberalisierung in Erbringungsart 4, da sie die Schnittmenge zwischen Migration und internationalem Handel berührt und deswegen Probleme aufwirft, die den Rahmen der Handelspolitik sprengen. Vielfach wird befürchtet, dass einzelstaatliche Gesetze und Vorschriften, die Einreise- und Aufenthaltsbestimmungen sowie Arbeit und Sozialversicherungsmaßnahmen regeln sollen, durch eine weitere Liberalisierung des Dienstleistungshandels in der WTO infrage gestellt werden könnten.

Die Gewerkschaften in der EU befürworten zwar die Freizügigkeit der Arbeitskräfte, argumentieren aber auch, dass die Liberalisierung der Erbringungsart 4 in ihrer aktuellen Form potenziell einer unkontrollierten Migration die Türen öffnen könne. Dies würde mit einer Belastung lokaler Beschäftigung, Löhne und Sozialstandards einhergehen. Ihre Bedenken hinsichtlich der Erbringungsart 4 betreffen vornehmlich die Migration von selbstständig tätigen Dienstleistern und Vertragsdienstleistern sowie denkbare Wanderungen von Dienstleistungserbringern mit geringen oder mittleren fachlichen Qualifikationen. Die Verhandlungen sollten den Schutz von Wanderarbeitern vor allen Formen der Diskriminierung garantieren und gewährleisten, dass die wichtigsten inter- nationalen Arbeitsnormen, das einzelstaatliche Arbeitsrecht und bestehende Tarifverträge anerkannt werden und dass die umfassende Einbindung der Internationalen Arbeitsorganisation (IAO) erfolgt (European Trade Unions Confederation 2005).

Obgleich es bereits eine ausgeprägte, länderübergreifende Spaltung zwischen Gegnern und Befürwortern der Dienstleistungsliberalisierung nach verschiedenen Interessengruppen gibt, lässt sich eine weitere Trennlinie zwischen verschiedenen Länderblöcken ausmachen. Diese verläuft zwischen den entwickelten Volkswirtschaften und den Entwicklungsländern. Während die EU ein hauptsächlich defensives Interesse an der Erbringungsart 4 hat, messen viele Entwicklungsländer ihrer weiteren Liberalisierung spezielle Bedeutung zu und halten diese für ein Kernelement der GATS-Verhandlungen innerhalb der Doha-Runde. Länder wie Indien verfügen über wettbewerbsfähige Exporteure von Computer- und Ingenieurleistungen und interessieren sich deshalb besonders für die Liberalisierung des Verkehrs von Dienstleistungserbringern in dieser Kategorie. Vor allem wollen die Entwicklungsländer die Erbringungsart 4 liberalisieren, weil sie über komparative Vorteile in arbeitsintensiven Dienstleistungen mit geringen und mittleren Qualifikationen verfügen, zum Beispiel im Verkehrswesen, Bauwesen und Fremdenverkehr, in den Pflegeberufen und bei Reinigungsdiensten.

\section{Ausmaß der Migration in der Erbringungsart 4}

Weil zuverlässige und vergleichbare internationale Statistiken fehlen, lässt sich nur schwer ermessen, inwieweit der Dienstleistungshandel über den Verkehr natürlicher Personen stattfindet. Dennoch haben einige wenige Studien versucht, den Wert des Handels in Erbringungsart 4 zu erfassen.

Diejenigen Studien, in denen die Vergütung ausländischer Zeitarbeiter zur Erfassung der Erbringungsart 4 verwendet wird, gehen davon aus, dass dieser Dienstleistungshandel 1997 mit 30 Mrd. USDollar zu beziffern war, was etwa 1,4 \% des Handels mit Dienstleistungen ausmachte. Demgegenüber trugen die Erbringungsarten 1 und 3 zusammen etwa $80 \%$ zum 
Dienstleistungshandel bei (Karsenty 2000). Jüngere Berechnungen durch das Sekretariat der Welthandelsorganisation stellten ebenfalls fest, dass die Erbringungsart 4 lediglich $1 \%$ des weltweiten Handels mit Dienstleistungen ausmache (WTO 2002).

Arbeitsmarkt- und Migrationsstatistiken könnten ebenfalls zugrunde gelegt werden, um sich einen ersten Überblick über das mögliche Ausmaß und die Bedeutung der Wanderungen unter der Erbringungsart 4 zu verschaffen. Schätzungen im Vereinigten Königreich, die sich auf die Anzahl der Arbeitsgenehmigungen für Nicht-EU-Arbeiter stützen, geben beispielsweise an, dass sich die Dienstleistungsimporte durch den Verkehr von Zeitarbeitern im Jahr 2000 auf knapp 2,5 Mrd. US-Dollar beliefen: Das entspricht 0,2\% des Bruttoinlandsproduktes (BIP) im Vereinigten Königreich (Gamberale 2006). Daten des Innenministeriums zeigen, dass die Anzahl der befristeten Arbeitsgenehmigungen im Dienstleistungssektor zwischen 1995 und 2000 um mehr als die Hälfte anstieg (auf 60.000 Genehmigungen), die meisten in den Sektoren Gesundheitsdienste ( $24 \%$ aller Genehmigungen), Computerdienste $(21 \%)$, Dienstleistungen für Geschäftsbetriebe (15\%) und Finanzdienstleistungen (12\%). Die Zuverlässigkeit dieser Daten zur Messung des Handelsaufkommens nach Erbringungsart 4 wird jedoch bezweifelt, weil sie auch den befristeten Verkehr von Arbeitern im Rahmen der bilateralen Programme umfassen. Selbstständig Tätige dagegen fallen nicht unter diese Arbeitsgenehmigungskategorie.

Die vorhandenen Statistiken mögen nicht ausreichen, doch deuten die bestehenden Daten darauf hin, dass die befristete Arbeitsmigration zunimmt. Die Arbeitsmobilität für Facharbeiter steigt ebenfalls an, häufig mit Förderung durch Sonderprogramme, und sie scheint sich auf die Dienstleistungssektoren zu konzentrieren. Jedoch erlauben bedauerlicherweise die Daten keine Antwort auf Fragen z. B. nach dem Ausmaß der Wanderungen von Dienstleistungserbringern in Modus 4 sowie nach ihren Qualifikationsniveaus und ihrer beruflichen Gliederung.

\section{Mögliche Auswirkungen auf die Aufnahmeländer}

\subsection{AUSWIRKUNGEN AUF DEN NETTO-WOHLSTANDSGEWINN}

Wirtschaftstheorien beschreiben den grenzübergreifenden Verkehr von Arbeitnehmern häufig aus Allokationsgründen als tendenziell vorteilhaft, zumindest für die Wirtschaft insgesamt. Dem liegt die Überlegung zugrunde, dass Migrationsprozesse Menschen von einem Ort, wo sie weniger produktiv sind, zu einem Ort hinführen, wo sie produktiver sind. Des Weiteren erhöhen ausländische Arbeitskräfte die Anzahl der inländischen Erwerbstätigen; sie steigern das Maß des Arbeitskräfteeinsatzes in der Volkswirtschaft und heben damit auch den potenziellen Arbeitsertrag.

Die Mehrzahl modellgestützter Studien, die darauf abzielen, die Auswirkungen der Liberalisierung im Dienstleistungshandel und damit auch die Folgen der Erbringungsart $4 \mathrm{zu}$ messen, konzentriert sich auf die erwarteten globalen Netto-Wohlstandsgewinne (Walmsley/Winters 2002). Schätzungen zufolge wird es sowohl für entwickelte Länder wie für Entwicklungsländer Zugewinne geben. Diese wären hauptsächlich zurückzuführen auf die Liberalisierung der Migration minder qualifizierter Arbeitskräfte aus den Entwicklungsländern (wo sie im Überangebot sind) in die entwickelten Länder (die relativ unterversorgt sind), und weniger auf die Liberalisierung der Migration hochqualifizierter Arbeitskräfte (Stiglitz/Charlton 2005).

Laut Schätzungen zu den wirtschaftlichen Folgen der Handelsliberalisierung liegen die globalen Jahresgewinne durch die Liberalisierung der Erbringungsart 4 zwischen 150 und 200 Mrd. US-Dollar (Walmsley/Winters 2002; Rodrik 2002). Die bekannteste und meistzitierte Forschungsarbeit zur Erbringungsart 4 im GATS von Winters et al. geht davon aus, dass der weltweite Wohlstandsgewinn um über 150 Mrd. US-Dollar pro Jahr ansteigen könnte, wenn die entwickelten Länder einen Anstieg der Zeitarbeiter aus Entwicklungsländern um $3 \%$ (ca. 8 Mio. Facharbeiter und 8,4 Mio. ungelernte Arbeitskräfte) zuließen. Für die EU würde sich der Netto-Wohlstandsgewinn auf
68,5 Mrd. US-Dollar beziffern (Winters et al. 2002).

Die Schätzungen weisen große Gesamtzugewinne auf. Erstens deshalb, weil bei den Unterschieden für Dienstleistungspreise und Löhne zwischen Industrie- und Entwicklungsländern ein Quotient von über zehn veranschlagt wird, wohingegen dieser Quotient im Warenhandel bei zwei liegt. Daher sind die erwarteten Gewinne durch die Liberalisierung des Dienstleistungshandels und damit auch des befristeten Verkehrs von Dienstleistungserbringern generell höher als die Gewinne durch die weitere Liberalisierung des Warenhandels (Rodrik 2002). Zweitens ist das Maß an Schutz in Verbindung mit dem Handel in Erbringungsart 4 im Dienstleistungssektor höher als für Warenmärkte. Dienstleistungen machen zudem einen großen und weiter zunehmenden Anteil des Welthandels aus. Demgemäß würde der Abbau von Beschränkungen im Verkehr von Dienstleistungserbringern zu signifikanten NettoWohlstandsgewinnen führen.

Studien, die Wohlstandsgewinne durch Handelsliberalisierung modellieren, werden von den Befürwortern einer raschen und extensiven Liberalisierung des Dienstleistungshandels in der WTO viel zitiert. Die Modelle und die Ergebnisse solcher Studien müssen jedoch kritisch infrage gestellt werden und sind mit Vorsicht einzusetzen (Taylor/Armin 2006; Stiglitz/Charlton 2006). Erstens sind die Ergebnisse von Modellen von ihren Annahmen abhängig. Im Falle von CGE-Modellen (berechenbare allgemeine Gleichgewichtsmodelle) sind diese restriktiv. Die Analyse beruht auf dem neo-klassischen Modell, das bei perfekten Marktbedingungen von Vollbeschäftigung, vollständiger Information, schneller Anpassung etc. ausgeht. Solche Annahmen sind für jedes Land fragwürdig, insbesondere für die Entwicklungsländer. Zweitens beschönigen auf CGE-Modellen basierende Analysen gerne die Tatsache, dass die Folgen in verschiedenen Ländern unterschiedlich ausfallen dürften und dass unterschiedliche Gruppen innerhalb der Länder auf unterschiedliche Art betroffen sein werden. Diese Studien lassen drittens die durch die Umsetzung von GATS-Bestimmungen erwachsenden Kosten außer Acht. Die hier skizzierten Einschränkungen gelten in noch größerem Ausmaß für die Studien, die Wohlstandsgewinne aus der Liberalisierung der Erbringungsart 4 modellieren, da sich die Mobilität von Arbeitskräf- 
ten an sich schon nicht für eine einfache Kosten-Nutzen-Analyse eignet, unter anderem wegen der vielen nicht messbaren Aspekte der Migration.

\subsection{HANDELSFOLGEN}

Die Liberalisierung der befristeten Migration von Dienstleistungserbringern hat Auswirkungen auf den Dienstleistungshandel in anderen Erbringungsarten und auf Handelsströme im Allgemeinen. Das Maß der Auswirkungen hängt davon ab, ob die Erbringungsart 4 andere Erbringungsarten ersetzt oder sie ergänzt, bzw. ob sie einfach die einzige verfügbare Erbringungsweise für die Dienstleistung ist.

Wenn eine Dienstleistung in verschiedenen Modi erbracht werden kann, dann könnte eine Liberalisierung in der Erbringungsart 4 zu einer Schwächung des Handels in anderen Erbringungsarten führen. Es gibt jedoch wichtige Verflechtungen zwischen der Erbringungsart 4 und anderen Modi des Dienstleistungshandels, und ein vermehrter Handel in der Erbringungsart 4 wird eher einen Handelsanstieg in anderen Versorgungsarten mit sich bringen (OECD 2003a). Die empirische Forschung zu einer Verbindung zwischen Immigration und Import- wie Exportvolumen hat gezeigt, dass ein $10 \%$ iger $\mathrm{Zu}$ wachs der Zahl von Wanderarbeitnehmern die Handelswarenexporte um 0,1-2,5\% ansteigen lässt, während die Wirkung auf Einfuhren zwischen $0,1 \%$ und $3,1 \%$ betrug (Jansen/Piermartini 2004).

Gleiches gilt für den Fall der Güterimporte: Eine gesteigerte Einfuhr von Dienstleistungen wird wahrscheinlich eine $\mathrm{He}$ rabsetzung der Inlandspreise für die jeweiligen Dienste bedingen. Für die Verbraucher und die Produzenten, die diese Dienstleistungen als Vorleistung verwenden, ist dieses ein Vorteil. Die Liberalisierung der Erbringungsart 4 könnte auch andere Folgen für den Handel haben. In der Literatur über Migration und Handel wird diskutiert, dass Wanderarbeitnehmer mit dem Aufbau von Netzen beginnen könnten, die für neue Geschäftschancen während ihres Aufenthalts im Aufnahmeland und auch danach genutzt werden könnten (WTO 2004). Darüber hinaus konsumieren Zeitarbeiter im Aufnahmeland einen Teil ihres Einkommens und machen Gebrauch von den Versorgungsleistungen dieses Landes; so tragen sie zu einer Steigerung der Nachfrage nach bestimmten Leis- tungen bei. Und schließlich kann der Aufenthalt von Dienstleistungserbringern im Ausland durch die vermehrte Nachfrage nach Auslandsprodukten auch den Warenhandel fördern.

\subsection{FOLGEN AM ARBEITSMARKT}

Das GATS betrifft lediglich die befristete Migration von Dienstleistungserbringern. Einerseits macht es der vorübergehende Charakter der Migration von Arbeitnehmern gemäß dem GATS einfacher, auf Arbeitskräftemangel in der EU zu reagieren. Andererseits sind die GATS-Verhandlungen so strukturiert, dass eine fortschreitende und kontinuierliche Liberalisierung gewährleistet ist, und die von Mitgliedern eingegangenen Verpflichtungen sind bindend. Doch Maßnahmen der Migrationspolitik werden für gewöhnlich als Antwort auf Arbeitsmarkterfordernisse ergriffen. Zwischen variierenden Arbeitsmarktanforderungen und dem dauerhaften Charakter der GATS-Verpflichtungen kann es deshalb zu Spannungen kommen.

\section{AUSWIRKUNGEN AUF DIE EINHEIMISCHEN ARBEITSPLÄTZE UND LÖHNE}

Befürworter der Liberalisierung von Erbringungsart 4 argumentieren, befristet anwesende ausländische Arbeitskräfte seien generell eher eine Ergänzung zu, als ein Ersatz für lokale Arbeitskräfte, und Zeitarbeiter würden nur in den Wirtschaftssektoren eingesetzt, in denen ein Arbeitskräftemangel herrsche. Doch auch das Gegenteil kann zutreffen, da Dienstleistungserbringer, die temporär nach Modus 4 migrieren, eine direkte Konkurrenz für Dienstleister in der EU sein können. Darüber hinaus ist es möglich, dass die wachsende Zahl solcher befristeten Aufenthalte und die kontinuierlichen Zuflüsse von Dienstleistungserbringern erheblichen Wettbewerb für lokale Arbeitskräfte erzeugen. Die unklaren Bestimmungen im GATS, die einen Einsatz von Arbeitskräften in der Erbringungsart 4 auf Rotationsbasis nicht verhindern, schüren solche Befürchtungen.

Die Auswirkungen auf Löhne sind dabei abhängig von der relativen Zusammensetzung der Qualifikationen bei ausländischen und einheimischen Dienstleistern. Studien zur Messung der Liberalisierungsfolgen in der Erbringungsart 4 auf die Löhne lokaler Arbeitnehmer stehen noch aus.
Den potenziellen Auswirkungen der Wanderarbeit auf Löhne in Aufnahmeländern wurde jedoch in den akademischen und politischen Debatten große Aufmerksamkeit gewidmet. Borjas weist in seiner Studie zu den Auswirkungen der Arbeitsmigration auf die Löhne in den USA beispielsweise nach, dass ein Einwanderungszuwachs um $10 \%$ die Einkommen der lokalen Arbeitnehmer zwischen 1980 und 2000 um 3-4\% minderte. Noch schwerwiegendere Folgen diagnostizierte er für lokale Arbeiter ohne eine Oberschulbildung (etwa $7,4 \%)$. Allerdings stellt er auch fest, dass die Hauptkonkurrenz für Immigranten am Arbeitsmarkt andere Immigranten sind (Borjas 2003). Longhi et al. ermitteln in ihrer Forschung zu Migrationsauswirkungen auf Löhne in den USA, Deutschland, den Niederlanden und Österreich, dass ein einprozentiger Zuwachs des Anteils an Immigranten in der erwerbstätigen Bevölkerung die Löhne um lediglich $0,1 \%$ verringert (Longhi et al. 2004). In Zukunft könnte die Verfügbarkeit ausländischer Zeitarbeiter allerdings auch den Druck reduzieren, den Arbeitskräftemangel durch Verbesserung der Lohn- und Arbeitsbedingungen zu beseitigen (Internationale Organisation für Migration 2003).

Das GATS könnte außerdem Folgen für das Ordnungs- und Vollstreckungswesen haben. Im Angebot der EU wird unterstrichen, dass die Gesetze und Vorschriften hinsichtlich Einreise, Aufenthalt, Arbeit und Sozialversicherungsmaßnahmen der EU und der Mitgliedstaaten für die Dienstleistungserbringung nach Modus 4 gelten, einschließlich aller Vorschriften bezüglich Aufenthaltsdauer, Mindestlohn sowie Tariflohnvereinbarungen (Europäische Kommission 2004). Viele Gewerkschaften haben jedoch Bedenken, dass die Liberalisierung in der Erbringungsart 4 zu Sozialdumping führen könnte und dass die schwer erkämpften Sozialnormen in der EU aufgeweicht werden. Die Gewerkschaften in der EU verlangen Gleichheit von Löhnen und Konditionen für aus- und inländische Arbeitnehmer (Waghorne 2003). In einigen Entwicklungsländern wird jedoch die Auffassung vertreten, dass die Lohngleichheit zwischen inländischen und ausländischen Arbeitnehmern die Nutzung ihres komparativen Vorteils behindere (OECD 2003a). Selbst dort, wo Gesetze zur Lohngleichheit durchgesetzt würden, ließen sich die Löhne, die den ausländischen Arbeitern gezahlt werden, nur schwer kon- 
trollieren. Bei der Durchsetzung könnten Schwierigkeiten auftreten, da Arbeitsgesetzgebung oder Tarifvereinbarungen bestimmte Arbeitnehmerkategorien im EUAngebot möglicherweise entweder gar nicht oder nicht in vollem Umfang erfassen, namentlich Nachwuchskräfte und Freiberufler (Union Network International 2005). Daher werden diverse Bedenken in Bezug auf die Durchsetzung von Arbeitsnormen angemeldet, denn das GATS sieht weder eine Definition noch den Schutz der Rechte der betroffenen Arbeitnehmer vor. Auf Zeit tätige Dienstleister werden wahrscheinlich eher schlechteren Arbeitsbedingungen ausgesetzt sein als reguläre Arbeitskräfte. Sie könnten aufgrund ihres befristeten Aufenthalts im Aufnahmeland auch eher gewillt sein, diese Konditionen zu akzeptieren (Raza 2003).

\section{GEWERKSCHAFTS- UND GESELLSCHAFTSPOLITISCHE KONSEQUENZEN}

Die gewerkschaftliche Vertretung temporärer ausländischer Dienstleistungserbringer ist aus mehreren Gründen schwieriger als bei fest angestellten Arbeitskräften: Einerseits sind sie nur kurzzeitig im jeweiligen Land, und andererseits üben die Arbeitgeber einen großen Druck auf sie aus, damit sie nicht in eine Gewerkschaft eintreten. Die zunehmende Liberalisierung in der Erbringungsart 4 und der mögliche Einsatz von Arbeitnehmern der Erbringungsart 4 als Ersatz für lokale Arbeitskräfte könnten deshalb einen tief greifenden Einfluss auf Tarifverhandlungen in der EU haben, da sich die Verhandlungsmacht bei Arbeitskonflikten und Lohnverhandlungen zugunsten der Arbeitgeber verlagern würde (Raza 2003).

Nicht zuletzt sind die Auswirkungen auf die Staatsfinanzen ein wichtiges Element in der Debatte um die Liberalisierung der Erbringungsart 4. Generell wird die temporäre Migration den Wohlfahrtsstaat in den Aufnahmeländern weniger unter Druck setzen als die permanente Einwanderung. Es fallen zum Beispiel geringere Kosten bei der Sozialversicherung und bei der sozialen Integration von ausländischen Arbeitskräften an, wenn diese nur vorübergehend in einem Land arbeiten. Dienstleistungsanbieter mit befristetem Aufenthalt lassen ihre Familien außerdem in der Regel häufiger in den Heimatstaaten zurück als dauerhafte Migranten und stellen damit geringere Ansprüche an soziale Wohlfahrtseinrichtungen.

Bei der temporären Migration in der Erbringungsart 4 treten jedoch Probleme auf, die mit den Unterschieden in den Sozialversicherungssystemen und -beiträgen in den Aufnahme- und Entsendeländern von Immigranten zusammenhängen. Werden beispielsweise Arbeiten an ein ausländisches Unternehmen untervergeben, so werden die Sozialversicherungsbeiträge für Projektarbeiter je nach den Bestimmungen im Heimatland der ausländischen Firma häufig durch diese bezahlt (Winters et al. 2002). Dazu gibt es eine ganze Bandbreite spezifischer Migrationsprobleme aufgrund der Erbringungsart 4 im GATS, so zum Beispiel das Überschreiten der Aufenthaltserlaubnis, mangelnde Achtung sozialer Rechte, kulturelle Integrations- sowie Sicherheitsprobleme. Einige Autoren argumentieren, dass ein gut geführtes Programm für befristete Mobilität die sozialen und politischen Kosten der dauerhaften Einwanderung teilweise verringern könnte, weil es der Bevölkerung vor Ort zeigen würde, dass eine Einwanderung nicht beabsichtigt ist (Winters 2005). Es könnte aber auch sein, dass die sozialen Anpassungskosten für Zeitarbeiter höher sind, da sie keinen Anlass haben, sich in das Gastland einzugliedern. Eine weitere Sorge ist, dass die temporäre Mobilität der erste Schritt hin zu einer dauerhaften Einwanderung sein könnte. Man kann vermuten, dass die Neigung ausländischer Dienstleistungsanbieter, dauerhaft in einem anderen Land zu leben, zumindest teilweise von der Art ihrer Arbeit abhängt - ob es sich also um eine Tätigkeit handelt, die an und für sich zeitlich begrenzt ist (Saisonarbeit, Arbeit an einem Bauvorhaben), oder um eine Tätigkeit unbefristeter Natur (Krankenpfleger, Computerspezialisten).

\section{Schlussfolgerungen}

Obwohl das GATS eine Übereinkunft zur Förderung und Stärkung des Dienstleistungshandels und keine internationale Migrationsvereinbarung ist, fällt eine Diskussion der Auswirkungen von Erbringungsart 4 ohne Einbeziehung der Migrationsprobleme schwer. Dabei unterscheiden sich die Methoden der WTO mit ihren Handelsrunden und unabänderlichen Abma- chungen sicherlich von den Migrationsverhandlungen.

Wie in den vorangegangenen Abschnitten dargestellt wurde, sind die GATS-Verpflichtungen und die Wanderungen von Dienstleistungserbringern in der Erbringungsart 4 bislang begrenzt. Wenig Personenverkehr in der Erbringungsart 4 des GATS bedeutet aber nicht, dass es keinen Verkehr von Zeitarbeitern gibt. Es existieren temporäre Programme und sie werden erweitert und verbessert. Das mag darauf hindeuten, dass es entweder keine Forderung nach multilateralen Eingaben zur Arbeitskräftemobilität gibt, oder aber, dass diese Forderung wohl besteht, dass aber das GATS sie nicht erfüllen kann. Man kann auch argumentieren, Arbeitsgenehmigungsfragen seien besser bilateral als multilateral zu lösen, da multilaterale Übereinkünfte zu wenig Spielraum böten. Solange der Umfang der Wanderungen temporärer Dienstleistungserbringer weiterhin nicht allzu groß ist, werden Negativwirkungen auf lokale Arbeitsmärkte eher begrenzt bleiben. Die Gesamtwirkungen der GATSbedingten Migration auf die Arbeitsmärkte könnten jedoch im Zusammenspiel mit allgemeineren Tendenzen zur internationalen Auslagerung und zum Einsatz von Vertragsarbeitern bedeutsamer werden. Natürlich ist es in der Praxis schwierig, diese Kausalketten voneinander zu trennen, und dies kann zu falschen „Schuldzuweisungen" führen.

Bei einem weiteren Wachstum des Handels mit Dienstleistungen könnte es notwendig sein, die Wanderungen der Dienstleistungsanbieter multilateral zu steuern. Und tatsächlich deutet sich ein Konsens darüber an, dass der rechtliche Rahmen für die Arbeitsmigration ausgeweitet werden sollte. Der eigentliche Punkt ist dabei aber nicht das Ausmaß des Nutzens, der generell von einer Liberalisierung der Erbringungsart 4 erwartet wird, sondern die Frage, welcher Nutzen garantiert werden kann und welche negativen Folgen minimiert werden können. Daher ist es dringend notwendig, die Liberalisierungsfolgen der Erbringungsart 4 weiter zu diskutieren, besonders angesichts der von den Gewerkschaften vorgebrachten Sorge um den Schutz der Sozialstandards in der Europäischen Union und um die Gleichbehandlung der Dienstleistungsanbieter.

Für eine akzeptable temporäre Migration bedarf es mehr, als die Beschränkun- 
gen grenzüberschreitender Mobilität abzusenken. Sie muss vielmehr auch in die Beschäftigungs- und Sozialpolitik integriert werden. Die Arbeitskräftewanderungen sind durch Arbeitsgesetzgebung und Gesetze zum Sozialschutz zu regulieren, und nicht (gewiss nicht ausschließlich) über
Handelsübereinkünfte. Eine isolierte Förderung der Arbeitskräftemigration durch die WTO birgt große Risiken - für die betroffenen Arbeitnehmer wie auch für die Legitimität der WTO selbst. Die Rolle der Sozialpartner und die Konsultation der IAO sind in diesem Verfahren von zen- traler Bedeutung, und die von zunehmenden Migrationsströmen betroffenen Gesellschaften müssen in Angelegenheiten der Liberalisierung der Erbringungsart 4 einbezogen werden.

\section{LITERATUR}

Borjas, G. (1995): The economic benefits from immigration, in: Journal of Economic Perspectives 2, pp.3-22

Borjas, G. (2003): The Labor Demand Curve is Downward Sloping: Reexamining the Impact of Immigration on the Labor Market, in: Quarterly Journal of Economics, November, pp. 1335-1374

Chanda, R. (1999): Movement of Natural Persons and Trade in Services: Liberalising Temporary Movement of Labour under the GATS, Neu-Delhi Europäische Kommission (2003): Antrag von WTO-Mitgliedern an die EG und ihre Mitgliedstaaten auf verbesserten Marktzugang für Dienstleistungen. Konsultationsdokument: http://europa.eu.int/comm/trade/ issues/sectoral/services/docs/imas.pdf

European Trade Union Confederation (2005): Position of ETUC on the 6th WTO Ministerial conference (13-18 December 2005, Hong Kong), Brüssel

European Services Forum (2005): Fourth ESF position paper on the temporary movement of persons, Brüssel

Gamberale, C. (2005): Presence of natural persons - Mode 4, New York International Organization for Migration (2003): World Migration 2003: Managing Migration Challenges and Responses for People on the Move, Band 2, World Migration Report Series, Genf

Jansen, M./Piermartini, R. (2004): The Impact of Mode 4 Liberalization on Merchandise Trade and on Other Modes of Trade in Services, Genf Karsenty, G. (2000): Assessing Trade in Services by Mode of Supply, in: Sauvé, P./Stern, R. M. (Eds.): GATS 2000: New Directions in Services Trade Liberalization, Washington D. C., pp. 33-56

Longhi, S./Nijkamp, P./Poot, J. (2004): A Meta-Analytic Assessment of the Effect of Immigration on Wages, Tinbergen Institute Discussion Paper OECD (2003a): Service Providers on the Move: Labour Mobility and the WTO General Agreement on Trade in Services, Grundsatzinformation, Paris
OECD (2003b): Service Providers on the Move: The Economic Impact of Mode 4, Working Party of the Trade Committee, Paris OECD (2003c): Bericht aus dem Seminar Handel und Migration, Paris Raza, W. (2003): Movement of Natural Persons (Mode 4) under GATS: an assessment of the current negotiations, BAK position paper Rodrik, D. (2002): Feasible Globalization. Centre for Economic Policy Research Discussion Papers, No. 3524, London Sinclair, S. (2006): Crunch Time in Geneva: pressure tactics in the GATS negotiations, Canadian Centre for Policy Alternatives

Stiglitz, J./Charlton, A.(2005): Fair Trade for All: How Trade Can Promote Development, New York

Taylor, L./Armin, R. (2006): Modelling the Impact of Trade Liberalisation, New York

Union Network International (2005): GATS Negotiations: EU Revised offer, Circular U055/GS

Waghorne, M. (2003): Mode 4 and Trade Union Concerns, in: Mattoo, A./Carzaniga, A.: Moving People to Deliver Services, Washington D. C. Walmsley, T./Winters, L. A. (2002): Relaxing restrictions on temporary movement of natural persons: a simulation analysis, Brighton Winters, L. A./Walmsley, T./Wang, Z./Grynberg, R. (2002): Negotiating the Liberalisation of the Temporary Movement of Natural Persons, Discussion Papers in Economics no. 87, Brighton Winters, L. A. (2005): Developing Country Proposals for the Liberalization of Movements of Natural Service Suppliers, Migration DRC Working Paper T8

WTO (2002): GATS, Mode 4 and the Pattern of Commitments, Genf WTO (2004): World Trade Report 2004, Selected Issues in Trade and Trade Policy, the liberalization of Services through the Temporary Movement of Natural Persons, Genf 